\title{
El Conquis: ¿un reality demasiado vasco? Análisis de una adaptación de un formato internacional
}

\author{
El Conquis: reality euskaldunegia? \\ Nazioarteko formatu baten egokitzapenaren analisia \\ El Conquis: a very Basque reality TV program? \\ Analysis of an international format's adaption
}

\author{
Andoni Iturbe Tolosa ${ }^{\star}$ Itxaso del Castillo Aira \\ Universidad del País Vasco (UPV/EHU)
}

\begin{abstract}
RESUMEN: Este trabajo de investigación se centra en el estudio de caso del reality El conquistador del fin del mundo, el espacio más exitoso de EiTB (grupo de radiotelevisión pública vasca) en los últimos quince años. La metodología ha consistido, por un lado, en el visionado completo de la edición 2018/19 (una de las más vistas de su historia) y en las entrevistas semiestructuradas con sus creadores. El objetivo del artículo es analizar y explicar la singularidad del formato, por lo que se ha optado por herramientas metodológicas que combinan los estudios antropológicos y televisivos, dentro del ámbito de los Estudios Culturales. Así, se han identificado algunas categorías que explican su excepcionalidad: la importancia y la tensión entre «jokoa» y «jolasa»; la estructura del grupo; la estrategia de los «zorros» y los «leones» y la representación de los cuerpos. La confluencia de dichas características determina un programa eminentemente vasco y singular que encarna la forma en que los vascos se ven y se representan.
\end{abstract}

PALABRAS CLAVE: Televisión pública; telerrealidad; docugame; formato; Survival.

ABSTRACT: This article focuses on the case study of the reality show El conquistador del fin del mundo, the most successful TV program broadcasted by EiTB (Basque public media group) in the last fifteen years. The objective of the article is to analyze and explain the singularity of this adaptation of an international format. The methodology consisted in the complete viewing of the edition $2018 / 19$ (one of the most viewed in its history) and semi-structured interviews with its creators. A mixed methodology was used, combining Anthropological and Television Studies, within the scope of Cultural Studies. Thus, some categories have been assigned in order to explain its exceptionality: the importance and tension between «jokoa" and «jolasa»; the structure of the group; the strategy of the "foxes" and the «lions» and the representation of the bodies. The confluence of these characteristics determines an eminently Basque and singular TV program, which embodied the way in which Basque society imagines and represents itself.

KEYWORDS: Public TV; Reality TV; Survival; Docugame; TV Format; Survival.

\footnotetext{
* Correspondencia a / Corresponding author: Andoni Iturbe Tolosa. Departamento de Comunicación Audiovisual. Facultad de Ciencias Sociales y de la Comunicación. Campus de Bizkaia de la UPV/EHU. Barrio Sarriena, s/n (48940 Leioa-Bizkaia) - andoni.iturbe@ehu. eus - https://orcid.org/0000-0002-1184-4634
}

Cómo citar / How to cite: Iturbe Tolosa, Andoni; Del Castillo Aira, Itxaso (2020). "El Conquis: ¿un reality demasiado vasco? Análisis de una adaptación de un formato internacional», Zer, 25(49), 153-169. (https://doi.org/10.1387/zer.21677).

Recibido: 25 abril, 2020; aceptado: 05 octubre, 2020.

ISSN 1137-1102 - eISSN 1989-631X / (C) 2020 UPV/EHU 


\section{Introducción}

El objeto de análisis de este artículo de investigación es El conquistador del fin del mundo (ETB2), un reality que se emite con éxito en la televisión pública vasca, en su canal en castellano, desde 2005. La decimoquinta edición (2018/2019) tuvo el mejor resultado de las siete ediciones anteriores y ha sido el tercero más eficaz en su historia en términos de audiencia (urbana y joven) y repercusión.

El conquistador del fin del mundo se ha convertido en una marca de la cadena junto a los informativos y las retransmisiones deportivas. A pesar de que no existen artículos de análisis científico previos, El Conquis (como se le conoce coloquialmente) es un programa de gran interés académico por varias razones. Primero, porque, en esa permanente connivencia del medio televisivo entre lo global y lo local, ha sabido adaptar un formato, el Survival, a las características de un territorio y lo ha hecho, además, exclusivo al mismo.

Segundo: por la naturalización de un espacio de telerrealidad en la parrilla de un canal autonómico público (ETB). Aunque no existe ninguna polémica actualmente sobre la idoneidad o no de la inclusión de ese formato en ETB2, los responsables del espacio no potencian los elementos más vinculados al reality y subrayan, en cambio, su carácter deportivo y competitivo para prevenir cualquier tipo de crítica.

Tercero: Lo que diferencia a El Conquis respecto a otros formatos, siempre basados en la competencia entre individuos, es la importancia del grupo. Los participantes de un focus group (Iturbe, 2012) definían El Conquis como una "competencia de grupos». Las categorías que derivan de ese estudio incidían en esa idea: «cercanía»; «nuestro»; "grupo». De hecho, una de los rasgos propios en los realities de ETB es la presencia del «nosotros» (el sentimiento de pertenencia a un grupo o estructura social) frente a la tendencia contemporánea hipertelevisiva (Iturbe 2012) del individuo en competición. EiTB tiene experiencia contrastada en la apuesta por «realities de grupo». Basetxea (2002-2008) fue el primer programa de telerrealidad de grupos de la historia del canal en euskera, ETB1.

Cuarto: por ir a contracorriente de la tendencia actual de la televisión social (Proulx y Shepatin, 2012) y de la televisión de proximidad (De Bustos y Casado, 2012) para crear comunidad, interacción y compromiso. En El Conquis no hay participación del público en el desarrollo del programa; es un espacio grabado, cerrado, que no se modifica por los votos o nominaciones del público. En ese sentido, no hace un uso estratégico de las redes sociales y de la comunicación digital para mejorar tanto el contenido, enriquecer la experiencia de la comunidad y crear eventos multimediales, hipertextuales e interactivos. Los creadores del formato se consideran de la «vieja escuela» de la televisión: no están pendientes de las narrativas transmedia actuales, por poner un ejemplo y entienden la profesión a la "vieja usanza». 
Quinta: pese a gozar de una gran reputación como espacio de aventuras duro, se trata de un programa que conecta con su territorio y con el que se identifica la sociedad vasca, hasta el punto de convertirse en un espacio dificilmente exportable. Cada vez que sus productores han intentado vender y promocionar El Conquis en otros mercados, ha sido rechazado por considerarse "demasiado vasco».

Sexta: La audiencia no para de crecer y cada año, a pesar de que la estructura se repite una y otra vez, se recogen mejores datos. El espacio no sufre el desgaste del formato y sus claves de éxito radican en códigos no sólo televisivos, tal y como veremos a continuación.

En el presente artículo se analizan los rasgos definitorios de El Conquis en relación a la comunidad que representa para explicar y dar con las claves de su éxito. Para ello, se apoya en una perspectiva antropológica, que tiene que ver con «el prisma desde el que muchos vascos se piensan y con la manera en que son pensados por otros» (González-Abrisketa, 2005:15).

\section{Marco teórico}

La literatura académica sobre la telerrealidad en España tuvo su apogeo a comienzos del siglo xxi bajo el paraguas del fenómeno Gran Hermano. Sin embargo, no existe un consenso sobre la etapa en la que nos encontramos debido a la complejidad del mapa audiovisual actual: la neotelevisión, nacida de la competencia que las cadenas de televisión privada; postelevisión (Imbert, 2008) y la hipertelevisión (Gordillo, 2009), en la que se ha querido aglutinar un estadio en el que entran en juego la publicidad y el entretenimiento, multiplicando los modelos híbridos ya surgidos en la neotelevisión (Gordillo et al., 2011). Asimismo, la reflexión académica sobre el docudrama resulta todavía muy escasa, en comparación con la atención que ha despertado en el mundo anglosajón (Raventós, Torregrosa y Cuevas, 2012), al igual que los términos género y formato. Las diferencias entre ambos se han difuminado (Puebla Martínez, Magro Vela y Fernández Valera, 2018:107). Destacan las aportaciones de Cascajosa (2007) que explica cómo la re-creación de la telerrealidad ha modificado la ficción, así como las claves de ficción que usan los realities para re-crear la realidad (Grela, López y Pérez Pereiro, 2018) y, de alguna manera, cómo este formato conecta con la audiencia más joven.

El formato Survival, en cambio, goza de un interés interdisciplinar renovado en la literatura académica anglosajona. Un reciente simposio de la Universidad de Sant Andrews (2019) analizaba las narrativas actuales del formato Survival desde una perspectiva transdisciplinar y de mutua influencia (videojuegos, películas y telerrealidad). Los formatos de televisión se comercializan internacionalmente y, hoy en día, todo 
el mundo, desde América a Asia, puede ver Big Brother, Got's Talent y otros espacios que presuponen una homogeneización en los gustos del espectador. La glocalización es uno de los rasgos definitorios de los formatos transcultural ${ }^{1}$ de ficción en televisión (García Avis, 2017) y de los formatos televisivos en general. «La dinámica más significativa parece ser la de adaptación, transferencia y reciclaje de narrativas y otros tipos de contenido» (Moran y Malbon, 2006:11).

El origen del El conquistador del fin del mundo es un espacio homónimo basado en un formato internacional producido por una productora argentina, Promofilm, filial de Globomedia (España). Se trataba de una coproducción entre cinco países: Brasil, EEUU, Chile, Ecuador y México. Razones coyunturales impidieron su continuación, pero Patxi Alonso, productor de Hostoil, pensó que podía interesar al público vasco.

El Conquis tiene rasgos del docugame clásico de supervivencia (Gordillo, 2013): espacio abierto y salvaje; condiciones duras; resistencia fisica y una emisión semanal a diferencia de la tónica internacional del formato Survival, que se centra en la evolución hipermoderna del individuo en condiciones extremas y sus correspondientes implicaciones ideológicas y sociales. En los últimos años se han publicado algunos trabajos que analizan la construcción de la masculinidad en el género (Champion, 2016), o la relación con el medio desde una perspectiva postcolonial (Rakesh, 2018).

\section{Metodología}

Los estudios televisivos (Television Studies) viven un nuevo florecimiento en el ámbito académico español y, tal y como sugiere el último gran libro sobre la programación televisiva española (Montero, 2018:17), se debe huir de la lacra de capturar el estereotipo (social, cultural, de género, de sexo, étnico, regional o «nacional»). Mediante una perspectiva interdisciplinar, el objetivo de esta investigación es identificar en el texto los elementos culturales y antropológicos vascos del formato y explicar así el éxito de El conquistador del fin del mundo, así como desvelar los otros elementos propios de la dinámica y práctica discursiva televisiva. Para ello, partimos de tres hipótesis:

- H1. Ante la tendencia global y transcultural del fenómeno Survival, las claves del éxito de El Conquis radican en la representación de ciertos valores culturales y antropológicos diferenciados que denominaremos como «vascos».

${ }^{1}$ La transculturalidad es una dinámica convergente entre lo global y lo local, según autores como Robertson. 
- H2 La práctica discursiva de El Conquis se basa en el concepto del juego como espacio de fuerza, astucia, sacrificio y comunidad mediante su encarnación en cuerpos fuertes, independientemente de su género.

- H3. La dialéctica de El Conquis bascula entre los valores tradicionales (lealtad, nobleza y sacrificio), la tensión entre el individuo y el grupo y los códigos televisivos propios del docugame contemporáneo.

Además del visionado completo de la decimoquinta temporada (2018-2019), hemos realizado una entrevista con Txema Montoya, uno de los creadores del formato junto a Joxean Goñi (director) y Patxi Alonso (productor y presentador de los debates). La entrevista ha aportado claves sustanciales para comprender la evolución del programa en sus 15 ediciones. Las entrevistas semiestructuradas son una herramienta de gran utilidad para examinar un producto cultural a través de la visión de sus creadores ya que revela el grado de premeditación y estrategia en el proceso de la práctica televisiva (preproducción, producción y posproducción) del formato.

Casetti y di Chio (1999) contabilizan once áreas diferentes de análisis televisivo. El presente se enmarca dentro del paradigma teórico de los estudios culturales (Cultural Studies), con su amplia atención al texto como a su contexto. Por todo ello, al entender que el análisis de contenido solo permitiría identificar ciertas categorías y una relación epidérmica, se ha optado por imbricar los estudios antropológicos en el análisis del formato televisivo. El objetivo no es, por tanto, analizar cómo se configura la identidad cultural vasca o se crean estereotipos, sino analizar y profundizar en los rasgos que, a través de un simple concurso, representan una manera de ser o de "ver» lo vasco. Solo así se entiende un programa tan imbricado en la parrilla y con un éxito sucesivo: hablamos de sustrato cultural, no de estereotipos.

Estos rasgos apelan al apego al grupo frente al individuo, a la honestidad encarnada en la fuerza y no en la inteligencia, y al sacrificio como valor máximo. Sin embargo, al tratarse de un programa de televisión, es decir, como realidad preconfigurada — pseudoevento tal y como decía Bort (2009)—, grabada de forma selectiva y ordenada de una manera intencionada, la realidad de los valores tradicionales vascos queda más en el terreno de la verosimilitud narrativa, ya que estos son incluidos en el relato para satisfacer el discurso en el que los vascos y vascas quieren verse representados.

\section{Mecánica del concurso}

La dinámica de El Conquis difiere en algunos pocos elementos del docugame de supervivencia. Se divide a los 33 concursantes en tres equipos, liderados 
por «capitanes» (personajes del deporte profesional que organizan a cada equipo para la competición, pero que no compiten para ganar). Cada equipo se mide en una prueba (la palabra que se utiliza es «juego»), que determina el tipo de campamento en el que habitarán: rico, pobre o muy pobre, lo que implica comida, agua y condiciones de pernocta muy extremas. Paralelamente, dentro de cada equipo se nominan para los duelos, del que saldrá el o la expulsada. Cuando los equipos se reducen, se unifican y empiezan a competir de forma «individual», pero siguen pensado en clave de grupo. Gana el grupo que vence en las distintas pruebas y juegos (no hay votaciones del público ya que está grabado) y el ganador último (individual) es aquel que consigue llegar primero a la última prueba, compuesta por seis finalistas.

La estructura de cada episodio se basa en el resumen de la convivencia, el anuncio y la preparación de la prueba, la prueba misma, la distribución de los nuevos campamentos, las nominaciones (que denominan «asamblea») y la inmunidad, y la prueba de los nominados. El equipo ganador celebra cada victoria ondeando la ikurriña (la bandera vasca). Pero la posee, finalmente, el o la ganador(a) del concurso, que también se lleva 20.000 euros.

Los concursantes de El Conquis solo habitan en condiciones ínfimas durante 25 días por lo que apenas hay un cambio físico evidente. Aun así, se autodefine como «el reality de superación más duro del mundo» en una triple dinámica: como juego, como competición y como aventura.

La edición del concurso del 2018/19 se grabó en Panamá. Se emitió los lunes y los miércoles se celebraron las galas en directo para comentar el concurso con los capitanes y los expulsados hasta el momento, y los familiares y amigos de los participantes. El equipo necesario para la realización del programa es de 150 personas, la mayoría dedicada a la grabación en ultramar.

\section{Análisis}

El espacio de telerrealidad ha sido el programa más seguido de la cadena durante la decimoquinta temporada, con una media de 144.000 espectadores, a los que habría que sumar la audiencia de Youtube y la opción «a la carta» (el servicio «nahieran») del ente público vasco de radiotelevisión. Además, lidera con rotundidad entre todos los targets y conecta especialmente con el segmento más deseado (25-44 años). Estos datos no siguen la estela de la cadena; al contrario, ETB ha descendido más de 10 puntos de share en los últimos 15 años, mientras El Conquis se ha mantenido o incluso ha aumentado sus datos. 
TABLA 1

Evolución de los datos de audiencia de El Conquis

\begin{tabular}{cccccccc}
\hline Temporada & Día & Hora & Emisiones & Duración & Rating & $\begin{array}{c}\text { Share } \\
\text { programa }\end{array}$ & $\begin{array}{c}\text { Share } \\
\text { medio de la } \\
\text { cadena en } \\
\text { temporada }\end{array}$ \\
\hline $2004 / 05$ & Lu & $22: 12$ & 14 & 106 & 7,0 & 17,6 & 18,7 \\
$2005 / 06$ & Lu & $22: 12$ & 14 & 126 & 6,6 & 16,4 & 16,6 \\
$2006 / 07$ & Lu & $22: 12$ & 16 & 112 & 7,3 & 16,9 & 14,9 \\
$2007 / 08$ & Lu & $22: 13$ & 16 & 118 & 8,2 & 19,5 & 14,8 \\
$2008 / 09$ & Lu & $22: 07$ & 17 & 123 & 9,4 & 22,3 & 14,7 \\
$2009 / 10$ & Do & $22: 11$ & 20 & 119 & 10,2 & 24,7 & 11,2 \\
$2010 / 11$ & Do & $22: 17$ & 20 & 130 & 8,1 & 20,1 & 8,3 \\
$2011 / 12$ & Do & $22: 19$ & 20 & 129 & 10,0 & 24,6 & 9,1 \\
$2012 / 13$ & Do & $22: 30$ & 23 & 136 & 7,6 & 19,4 & 10,0 \\
$2013 / 14$ & $\mathrm{Sa} / \mathrm{Do}$ & $22: 27$ & 37 & 81 & 6,3 & 15,4 & 9,3 \\
$2014 / 15$ & $\mathrm{Lu}-\mathrm{Do}$ & $22: 35$ & 27 & 101 & 5,1 & 13,0 & 8,4 \\
$2015 / 16$ & $\mathrm{Lu}$ & $22: 32$ & 19 & 122 & 5,1 & 14,4 & 7,4 \\
$2016 / 17$ & $\mathrm{Lu}$ & $22: 37$ & 20 & 122 & 5,6 & 16,6 & 7,6 \\
$2017 / 18$ & $\mathrm{Lu}$ & $22: 38$ & 20 & 127 & 5,3 & 16,3 & 8,3 \\
$2018 / 19$ & $\mathrm{Lu}$ & $22: 42$ & 19 & 121 & 6,8 & 21,9 & 8,2 \\
\hline
\end{tabular}

El Conquis tuvo una audiencia media de 21,9\% y la media de ETB2 fue de 8,2\% en la edición 2018/19.

Fuente: elaboración propia, partiendo de los datos facilitados por EiTB-Geca.

Un estudio reciente del proyecto Ikusiker (marzo, 2019) sobre los hábitos de consumo de los estudiantes universitarios vascos sostiene que el 28,69\% de los encuestados señala haber visto el programa, en un momento en el que los jóvenes no ven la televisión tradicional.

Tras el visionado de todos los capítulos de la edición 18/19 se detectaron algunos ítems: por un lado, lo tangible y/o lo visible (el valor material de las pruebas); por otro lado, los protagonistas (los concursantes) y, por último, la representación, el discurso y los códigos televisivos. Finalmente, tras una sistematización y categorización del contenido extraído, serán cuatro las categorías analizadas: la dialéctica entre jokoa y jolasa; la estructura grupal frente a la individual; la tensión entre ser «león» o ser «zorro» y la representación del cuerpo. 
TABLA 2

Categorías del análisis extraídas tras el visionado

\begin{tabular}{llll}
\hline \multicolumn{1}{c}{ Televisión } & \multicolumn{1}{c}{ Estructura } & \multicolumn{1}{c}{ Estrategias } & \multicolumn{1}{c}{ Discurso } \\
\hline Formato & Grupo & Leones & Cuerpo \\
Presentador & Individuo & Zorros & Representación \\
Pruebas & Convivencia & Conflicto & Masculinidad \\
\hline
\end{tabular}

Fuente: elaboración propia.

\section{1. «JOKOA ETA JOLASA»}

Tal y como plantea la antropóloga Olatz González-Abrisketa en sus escritos sobre la pelota $(2005 ; 2013)$, el juego es donde el imaginario de la sociedad que lo practica se reproduce y regenera. Los juegos de El Conquis sustentan y reproducen unos valores tradicionales de la comunidad vasca. De hecho, a las pruebas se les denomina, precisamente, «juegos» y sería la traducción de jokoa, un concepto fundamental, diferente del jolasa, otro tipo de juego. El antropólogo Joseba Zulaika (1991) distingue ambos: «jolasa» es el juego simbólico, pero también conversación, palabra, diversión o recreo; jokoa, en cambio, es el juego de competición. El primero es de mujeres o niños (jolas, padeia, play), mientras el segundo pertenece al ámbito público y masculino (joko, ludus, game). No es un término baladí ya que «la definición cultural de hacerse adulto es precisamente la capacidad de interiorizar el esquema de joko y operar con él» (Zulaika, 1991:207), así como la posibilidad de perder, entender el «joko» y ser jugador-jokalari (González-Abrisketa, 2005: 222).

El juego (duelos, pruebas) requiere de valores intangibles como la persistencia, la resistencia, el sacrificio o la fuerza («indarra», en euskera) que representan los concursantes («jokalari»). Otra vez remitiendo al euskera, se utiliza «indarrean» para decir algo que está vigente. El carácter «jokalari» y/o competitivo de El Conquis es una de sus señas de identidad. Por otro lado, bajo el concepto de «jolasa», se despliegan los elementos más televisivos: problemas de la convivencia, broncas, nominaciones, estrategia; mientras las pruebas físicas pertenecen al «jokoa». Las tensiones entre ambos tipos de juego se articulan en torno a la aceptación y recreación de unos valores y a la aceptación y disfrute de los valores propios del entretenimiento televisivo, basado en el conflicto.

El juego para ser tal debe tener su lugar entre iguales, del mismo nivel. El programa rompe esa regla en pos del espectáculo televisivo. La inclusión de personajes conflictivos o físicamente inferiores hace que, por un lado, el grupo deba protegerlos para poder sumar fuerzas y ganar cada juego y, por el otro, debe librarse de ellos 
para mantener un buen ambiente en la convivencia. La tensión entre el interés individual y grupal es permanente, y mueve las acciones de sus actores.

Esta desigualdad está planificada desde el casting. El casting es un componente primordial de la preproducción de un programa de televisión y parte del éxito de los programas. Los concursantes nutren la narración dando verosimilitud al relato con su personalidad individual y rol en el grupo, y es por esta razón por la que el casting se entiende como factor vital en formatos de telerrealidad (Puebla Martínez, Magro Vela y Fernández Valera, 2018:115). Los castings también sirven para establecer y marcar desde el inicio un tipo de relato y arquitectura narrativa. Además, la posproducción es uno de los elementos clave de la televisión actual.

Tal y como confiesa Txema Montoya, de los 33 participantes, tiene que haber 11 atletas, muy completos físicamente. Otros 10-11 personajes deben ser ambivalentes y deportistas, pero expresivos. Y otros 10-11, o puede que menos, que generen mucho ruido, capaces de provocar situaciones de conflicto.

Frente al ideal de lo "vasco», en el que la contención, el pudor y la honestidad son valores que se reclaman como propios del juego, el programa de televisión, como es propio en su naturaleza, busca permanentemente el conflicto como elemento catalizador del relato. La dirección del programa no puede guionizar el espacio, por lo que intenta provocar situaciones de las que espera una determinada trama. Tal y como comentan los creadores, la dirección del programa busca varios elementos que le reportan las claves del éxito. Por un lado, el conflicto que se vehicula siempre en las tensiones entre grupo e individuo: el relato de la amistad (la lealtad) y la traición (votar a uno/a del grupo); es decir, la trama que el guionista John Truby definía de "Aliado impostor» (Truby, 2009), por la que un supuesto aliado se revela como adversario de forma insospechada. No existe guion, pero se provocan situaciones: el éxito radica en que funcionen.

\subsection{Estructura GRUPAL FRENTE AL INDIVIDUAL}

La tensión entre el grupo y el individuo es uno de sus rasgos definitorios. Una vez que oficialmente se rompen y se unifican los grupos, se mantiene la lealtad hacia el grupo al que se ha pertenecido. Por tanto, cuando el presentador anuncia en el episodio 17 que siguen "16 aventureros por sí solos en la lucha fratricida», la realidad es que los concursantes siguen pensando en clave de pertenencia al equipo. En el siguiente episodio, el $18 .^{\circ}$, pese a que el presentador insiste en que ya no existen los grupos como tales, los concursantes tienden a verse como integrantes del mismo. "Yo soy azul y moriré azul», dice Luisito, uno de los finalistas sorpresa de esa edición. Según los creadores, la tendencia a verse como miembro de un equipo hasta el final tiene esa lectura positiva: el sentimiento de cuadrilla, de integración, que mu- 
chos lo mantienen hasta el final. «Cuando llega la unificación, deberían pelear cada uno por su cuenta, pero nos hemos fijado que siempre hay grupúsculos a los que le interesa permanecer juntos: por intereses y por afinidad, de forma natural. Porque se hacen muy amigos, hasta amigos para toda la vida», sostiene Montoya.

\subsection{Zorro y león. FuerZa y nobleza}

Hay dos formas de competir en un juego: con astucia o con fuerza. En El Conquis son muy conscientes de esta tipología y está presente en las dinámicas de juego del formato. Las pruebas físicas varían para dar cabida a todo tipo de jugadores: a los leones, pero también a los zorros. La fuerza no es siempre imprescindible para ganar. Sin embargo, se considera más noble que la astucia en el imaginario vasco. «La astucia no es un arma tan pura (...) Los zorros, que dominan a sus oponentes escondiendo sus armas, están siempre bajo sospecha. La fuerza, por el contrario, se considera honesta, transparente, noble. No hay nada sucio detrás de la fuerza. Es obvia» (González-Abrisketa, 2013: 96). Dos ejemplos ponen en evidencia este axioma: en el episodio 19, los concursantes tenían que encontrar unas conchas, abrirlas y esperar si contenían o no perlas. El grupo ganador (dos azules y una verde) fueron astutos y capaces de encontrar conchas arrinconadas y escoradas y fueron los ganadores sorpresa, pese a que fueron los que menos conchas recopilaron. "Que se valoren cuántas conchas se cogen, no cuántas llevaban perlas", lamentaban los «leones». Los lamentos de los «leones» fueron a más: «Esto no es El Conquis Casino»; "Nos preguntaron sobre nuestras capacidades para poder competir aquí», lamentaron.

En el final de El Conquis, uno de los finalistas, Jontxu, un «león» fortachón noble, no fue lo suficientemente «zorro» y se quedó fuera de la competición por dejar pasar a un compañero (Luisito, un "zorro») en la prueba de la escalada, y se quedó sin cuerda ni final.

Los valores del ideal vasco que deben reproducirse en el juego son la fuerza, la resistencia y la nobleza. Sin embargo, el programa, como reality, busca otro tipo de comportamiento en sus concursantes: fuerte pero individualista, que engañe y manipule a sus compañeros. Tal y como lo explicaba Montoya: «A nosotros los personajes que nos seducen son los otros, y si encima se ven capaces de ganar, eso es lo mejor. Porque si eres un personaje retorcido o que no gusta al grupo, pero que luego no te puedes defender en las pruebas fisicas y te vas a ir en la primera de cambio, te habrá solucionado un programa o dos, pero si encima pueden ganar y son fuertes fisicamente y retorcidos, es maravilloso». Mientras los concursantes y el público esperan ver ratificados sus valores respecto al juego, y, por tanto, su idea de «lo vasco", la dirección del programa potencia los conflictos y los participantes zorros para que esos valores se vean amenazados, generando emoción, interés y polémica. 
La dirección del programa trabaja para desmontar en las «asambleas» (nominaciones) el «intento de los concursantes de demostrar que todo va bien por vergüenza, algo muy vasco; quieren que la cámara no esté presente» y es el presentador quien les interroga de forma insistente hasta encontrar un resquicio de problema que consigue hacer presente y lograr que influya en la dinámica del concurso.

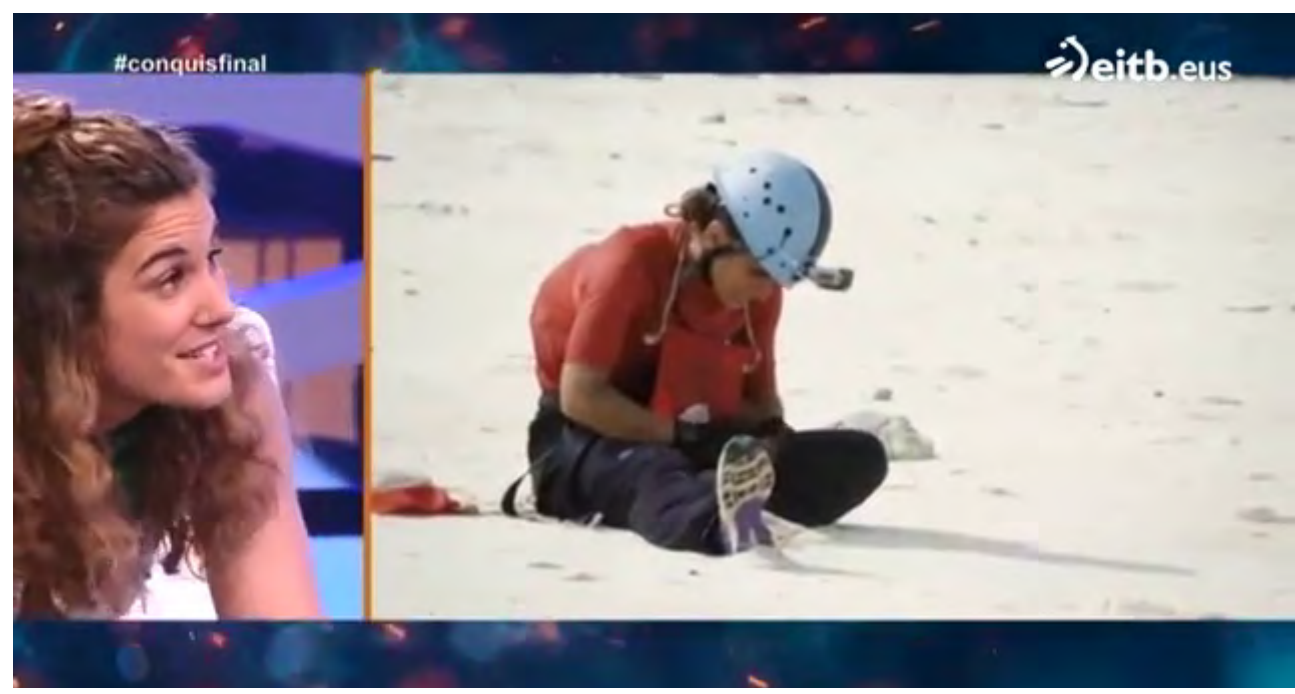

FigURA 1

En la imagen aparece Ianire, durante la final. Fue la única finalista de la edición 2018/19.

Pensó en continuar durante la final pese al dolor y una lesión manifiesta. La única finalista mujer de su edición se lesionó el tobillo y no pudo seguir las pruebas, pese a haberlo intentando en la final. En la imagen comenta el dolor y la pena por no haber podido seguir y competir en la final. Tuvo que abandonar la final

Paradójicamente, y contra la dinámica de su naturaleza televisiva, a la gente le gusta el programa por su «autenticidad». Según se recogía en un DAFO (Fortalezas, Oportunidades, Debilidades, Amenazas) encargado por la cadena en 2016, la percepción del programa hacía que se viera como un espacio auténtico (un término problemático para referirse a la telerrealidad, según Randall y Stacy, 2005) y que era muy duro, que no les daban comida por debajo u otro tipo de triquiñuelas que ellos sospechan que ocurre en otros programas similares. Sin embargo, se trata de una aventura que apenas llega al mes, por lo que la supervivencia no está en juego como tal, y pueden aguantar las malas condiciones durante ese periodo. En cambio, los juegos sí son especialmente duros. Y la forma en que los concursantes se enfrentan a ellos es, sobre todo, a través del sacrificio. Como mantiene González-Abrisketa, «el sufrimiento se considera el itinerario ideal para la constitución honesta y noble de la persona» (González-Abrisketa, 2005: 308). Las muestras del mismo han tenido lugar 
repetidas veces a lo largo de esta temporada: los concursantes han participado con lesiones, las han sufrido durante la competición y han continuado a pesar del dolor. Son sacrificios que se exigen al vasco en el juego, por la cualidad de nobleza que se atribuye y que constituye, en definitiva, la forma en que al vasco, tanto mujeres como hombres, le gusta representarse.

El ganador del concurso fue Iker Babio, calificado de «zorro» o «estratega» por sus compañeros y compañeras. La representación de su victoria se realizó en directo, en los estudios Miramón de ETB. La representación de la final fue comedida y modesta y contradiciendo a los códigos televisivos espectaculares de exceso (Deery, 2009); es decir, no fue apoteósica. Esta estética contenida hace referencia a los valores que, según la sociedad vasca, debe cumplir un ganador, en la que la austeridad de la emoción va vinculada con el sacrificio y la nobleza.

\subsection{Cuerpos masculinizados y una nueva masculinidad}

El deporte, tanto vasco como internacional, se asocia tradicionalmente con lo masculino. En concreto, los protagonistas de los juegos vascos son hombres. Es el cuerpo masculino en el que se identifica la comunidad imaginada vasca, excluyendo así a las mujeres de los acontecimientos deportivos y culturales públicos. El cuerpo de la mujer no puede encarnar a la comunidad más allá de como cuerpo maternal. «Ese imaginario en el que el cuerpo de la nación es susceptible de abstraerse exclusivamente a través de un cuerpo varón, un cuerpo que por su acomodo a dicho imaginario es capaz de atraer a otros cuerpos y encarnar así a la comunidad» (GonzálezAbrisketa, 2013: 93-94). El Conquis, sin embargo, ha incluido a las mujeres en el concurso desde sus inicios.

Su inclusión genera una serie de dinámicas. Por un lado, la igualdad de oportunidades que se le presupone al juego, se puede ver afectada por la supuesta superioridad física de los varones. Por otro lado, los cuerpos femeninos no son aptos para la identificación de la comunidad. Por ello, los cuerpos de las mujeres del programa se masculinizan, son cuerpos fuertes que encarnan ese valor comunitario central y así se presentan ellas: como deportistas, como agentes, sujetos y nunca como objetos de contemplación o de deseo.

El fisico musculado de buena parte de las concursantes remite a prácticas consideradas masculinas en la concepción heteropatriarcal, "que hacen visible un rechazo voluntario a la inactividad femenina», tal y como decía Halberstam (1998: 58), y que cuestiona el supuesto orden «natural» del cuerpo femenino, que es la debilidad. Su exhibición se atribuye a una subjetividad no heteronormativa. La fuerza no es un rasgo femenino y cuestiona el binario de los géneros. «La asociación cultural entre deportividad y actividad y masculinidad es una pareja ideológica que alienta 
la reticencia de mujeres y jóvenes a desviarse de los estándares sociales de ser activas y fuertes» (Clark, 2008:40). Las mujeres que acuden al programa son, en su mayoría deportistas, y sus cuerpos resultan casi indistinguibles de sus compañeros masculinos; no hay una cámara cosificadora ni una representación de hiperfeminidad.
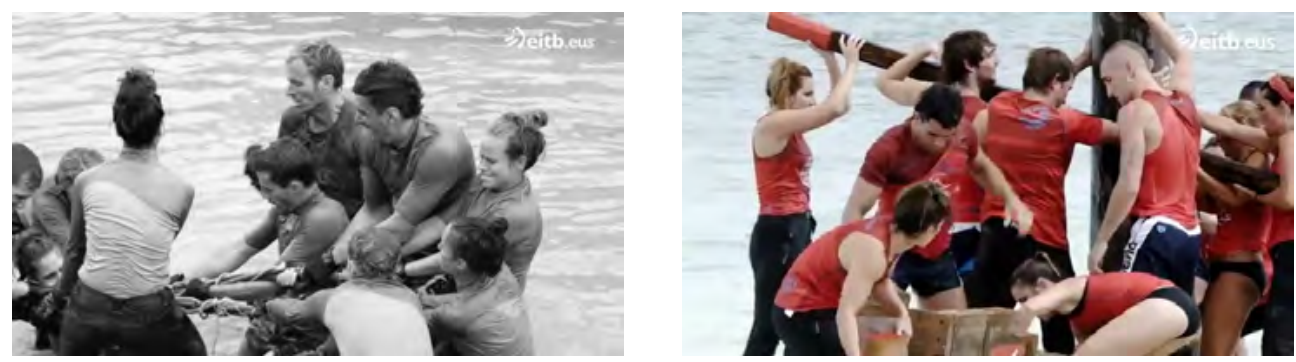

Figuras 2 y 3

Dos ejemplos de la convivencia y equiparación de los cuerpos masculinizados

En este sentido, el programa ha sido pionero al colocar a las mujeres en «la plaza», el espacio público. Las pocas mujeres pelotaris que históricamente han existido, así como las remeras o incluso las futbolistas, nunca han sido objeto de contemplación, no han supuesto un acontecimiento deportivo lo suficientemente valorado como para que existiera un público. Desde hace apenas 3-4 años, esta situación está cambiando y se han creado ligas de remo y pelota femenina, y las ligas de fútbol femenino empiezan a congregar a multitud de espectadores. Se verifica, por lo tanto, un cambio social respecto a la relación mujer, deporte y comunidad. En esta última edición de El Conquis, las dos capitanas del equipo más potente fisicamente, el equipo verde, eran una pelotari y una patrona de trainera, dos disciplinas que requieren fortaleza, sacrificio y bravura.
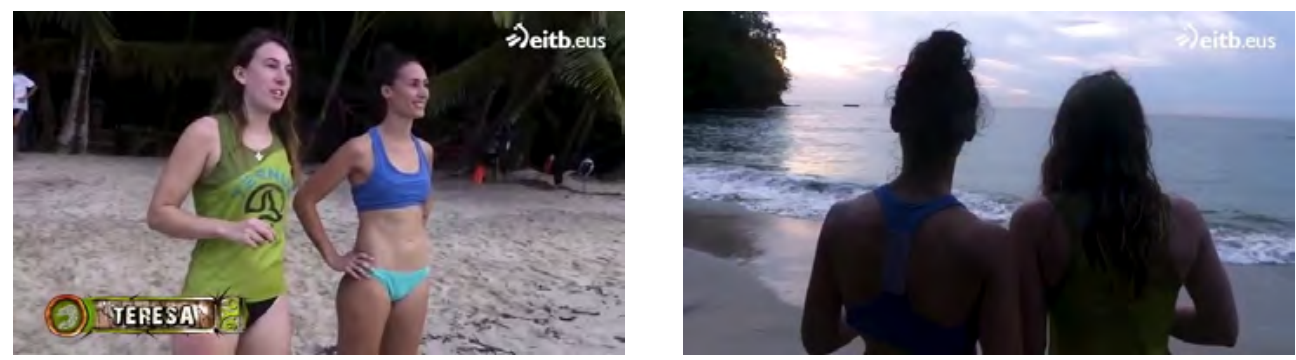

Figuras 4 y 5

Se trata de uno de los pocos travellings circulares de la última edición, que gira en torno al cuerpo. La cámara recorre sus cuerpos. Comienza con un plano medio en la que se resalta la postura y el lenguaje corporal (manos en la cintura, que reflejan seguridad y desafio), para después pasar un plano americano para terminar, a contraluz, con el horizonte en primer término 
Asimismo, la masculinidad que los programas Survival tienden a mostrar, esto es, cuasi militar, homofóbica y sin un lugar igualitario para las mujeres, está cambiando. Según Jared Champion (2016), el formato permite la inclusión de homosexuales, mujeres e incluso una presentación burlesca de la hipermasculinidad. Además de la inclusión igualitaria (o casi) de las mujeres en El Conquis, también asistimos en esta edición a la participación de un chico gay (Luisito) que hizo gala de su condición llevando unos tacones rojos al campamento. A pesar de no ser un hombre fuerte, consiguió, gracias a su astucia, llegar a la final.

\section{Conclusiones}

A través del escrutinio sobre el El Conquis se ha puesto en valor la singularidad de un formato imbricado en la parrilla del canal autonómico vasco (ETB2). El éxito manifiesto de la audiencia (un 22\% de cuota) pone de relieve la consolidación de un formato a lo largo de 15 años. La edición 2018/19 ha sido la tercera más vista de su historia y ha sabido segmentar y potenciar los targets de su audiencia. Queda demostrado que no se trata de un reality al uso, sino que activa una serie de procedimientos y prácticas discursivas sobre cómo es representado y visto lo vasco. Esa representación tiene lugar a través del juego. Dada su capacidad de generar emociones, los deportes son metáforas de las sociedades que los practican. El escenario de juego es el lugar donde ese imaginario se reproduce y regenera.

El Conquis reproduce y regenera ciertos valores vascos como el sacrificio, la fuerza, la nobleza y la importancia del grupo frente al individuo. La audiencia y el éxito del formato legitiman esta forma de verse y representarse lo vasco.

Asimismo, El Conquis es un programa de televisión, y como tal, se basa en el conflicto para generar interés. Aprovecha las tensiones que pueden tener lugar alrededor de esos valores para crear espectáculo. El concurso tiene dos partes diferenciadas: la convivencia y la prueba fisica, que en el argot del programa se llama «juego». La primera se englobaría bajo el concepto de «jolasa», en el que se despliegan los elementos más televisivos: problemas de la convivencia, broncas, nominaciones, estrategia... es decir, apelan a la astucia (el zorro); mientras las pruebas físicas pertenecen al «jokoa», que requieren fuerza (apelan a la fábula del «león»). Ambos tipos de juego requieren sacrificio; el camino hacia la nobleza.

Estos valores propios del «jokoa» son habitualmente adscritos a los hombres, actores tradicionales del deporte, pero en la actualidad las mujeres están ocupando un lugar en lo deportivo y, desde los comienzos del programa, han competido en igualdad de número, resultando cuatro veces ganadoras. Aunque la mujer sea admitida legítimamente como portadora de estos valores, se la representa, a cambio, masculinizada, y su cuerpo resulta casi indistinguibles de sus compañeros masculi- 
nos. Se trata de una representación de cuerpos sujeto; cuerpos-fuerza, en acción. En términos de la elección del encuadre de la cámara es fija, mayoritariamente frontal o móvil (travelling de seguimiento). No hay lugar para el hedonismo y la contemplación del cuerpo; sólo el sacrificio, la fuerza, la astucia, el grupo y la victoria.

Otro elemento susceptible es la importancia del grupo sobre el individuo, ya que genera conflictos y tramas narrativas. La estructura del grupo sobre el individuo rompe con la tendencia actual de la telerrealidad y el docugame contemporáneo y pone el foco en la representación de una comunidad imaginada a la hora de verse y representarse como vasca.

La intencionalidad simbólica del uso de la ikurriña (el triunfo final) a lo largo de sus quince ediciones ha pasado a formar parte de un ritual en la última edición (cada victoria de un juego se celebra con una ikurriña que ondea un capitán o una capitana). Esa ikurriña fue entregada por el lehendakari Ibarretxe al presentador Julian Iantzi en la primera edición que tenía lugar en la Patagonia. El acto fue improvisado, fruto de la casualidad, pero no deja de tener una significación simbólica poderosa que los responsables del programa le pidieran ese gesto al exjefe del Ejecutivo Vasco.

Es el ganador final, sin embargo, quien alcanza a poseer, no sólo ondear, la ikurriña. La puesta en escena de esa victoria final se realiza, a pesar de ser un programa de televisión, sin espectacularidad, con contención, que pone de manifiesto la especificidad de los discursos y prácticas que moviliza El Conquis en cuanto a representación y legitimación.

\section{Referencias bibliográficas}

Bort Gual, I. (2009). La Granhermanización de la Información Televisiva: asimilación de los estilemas del reality-show en los procesos del periodismo informativo (Actas del II Congreso Internacional de Teoría y Técnica de los Medios Audiovisua-

les) Universitat Jaume I, Castellón. Recuperado en: http://repositori.uji.es/xmlui/ handle/10234/34026

Casetti, F., Di Chio, F. (1999). Análisis de la televisión: instrumentos, métodos y prácticas de investigación. Barcelona: Paidós.

Cascajosa, C. (2007). «Reality Bites». De cómo la telerrealidad ayudó a salvar la ficción. Trípodos, 21, 97-102.

Champion, J. (2016). Survivor Shows and Caveman Masculinity» (2016). The Popular Culture Studies Journal, 240-258. https://mpcaaca.org/wp-content/uploads/2016/10/ PCSJ_v4_12.pdf\#page $=250$ 
Clark, E. (2008). Hairy Thuggish Women: Female Werewolves, Gender, and the HopedFor Monster. Washington: Universidad de Georgetown. https://repository.library. georgetown.edu/handle/10822/551562

De Bustos, J.C., Casado, M. A. (2012). Televisiones autonómicas: Evolución y crisis del modelo público de proximidad. Barcelona: Gedisa.

Deery, J. (2004). Reality TV as Advertainment. Popular Communication. 2:1, 1-20. < http:// dx.doi.org/10.1207/s15405710pc0201_1>

García Avis, I. (2017). La glocalización como rasgo definitorio del remake transcultural en televisión. Fonseca, Journal of Communication, 14, 91-111. <http://dx.doi. org/10.14201/fjc20171491111>

Gordillo, I., Guarinos, V., Checa, A., Alvarado, M. del Mar, Varea, J., López-Rodríguez, F., DE LOS SANTOS, F., Pérez-Gómez, M (2011). Hibridaciones de la hipertelevisión: información y entretenimiento en los modelos de infoentertainment. Revista Comunicación, 9, (1), 93-106.

Gordillo, I. (2009). Manual de Narrativa Televisiva. Madrid: Síntesis.

Gónzalez-Abrisketa, O. (2005). Pelota vasca: un ritual, una estética. Bilbo: Muelle de Uribitarte Editores.

Gónzalez-Abrisketa, O. (2013). Cuerpos desplazados. Género, deporte y protagonismo cultural en la plaza vasca. AIBR: Revista de Antropología Iberoamericana, 1, 83-110.

Grela., P; López X. y Pérez Pereiro, M. (2018). La re-creación de la realidad en los «reality shows». Revista de la Asociación Española de Investigación de la Comunicación, 5, 104-111. https://doi.org/10.24137/raeic.5.9.12

Halberstam, J. (1998). Female Masculinity. Durham: Duke University Press.

Ikusiker (2019). https://ikusiker.eus/wp-content/uploads/2020/02/1_ES_INFORME.pdf

Imbert, G. (2008). El transformismo televisivo. Postelevisión e imaginarios sociales. Madrid: Cátedra.

Iturbe, A. (2012). Representaciones del yo y del nosotros en los docudramas de ETB. Entre el servicio público y el prime time. En Crisis y políticas. La radiotelevisión en el punto de mira. Leioa: UPV/EHU (actas), 601-615.

Montero, J. (2018). Una televisión con dos cadenas. La programación en España (1956-1990). Madrid: Cátedra. Signo e imagen.

Puebla-Martínez, B.; Magro Vela, S. y Fernández Valera J. (2018). Funcionalidad de los componentes narrativos cinematográficos en los nuevos formatos televisivos: Los dating shows. Anàlisi: Quaderns de Comunicació i Cultura, 59, 105-119. doi https://doi. org $/ 10.5565 / \mathrm{rev} /$ analisi.3113

Moran, A.; Malbon, J. (2006). Understanding the global TV format. Bristol: Intellect Books. New Jersey: John Wiley\&Sons.

Proulx, M.; Shepatin, S. (2012). Social Tv: how marketers can reach and engage audiences by connecting television to the web, social media, and mobile. Hoboken, N.J.: John Wiley \& Sons. 
Rakesh, N. (2018). Relooking at neo-imperial ideologies in reality television series-man v. wild. Mass communicator: International Journal of Communication Studies, 12, 27-31. http://www.indianjournals.com/ijor.aspx?target $=$ ijor: mcomm\&volume $=12 \&$ issue $=3$ \&article $=004$

Raventós, C., Torregrosa, M, Cuevas, E (2012). El docudrama contempóraneo: rasgos configuradores. Trípodos, 29, 117-132.

Randall, R., Stacy L. W. (2005). Paradox and the Consumption of Authenticity through Reality Television. Journal of Consumer Research, 32, 284-296. https://doi. org/10.1086/432238

Truby, J. (2007). Anatomía del Guión. Barcelona: Alba Editorial.

Zulaika, J. (1991). Violencia Vasca: Metáfora y sacramento. Madrid: Nerea. 